\title{
Quantum chaotic scattering in graphene systems in the absence of invariant classical dynamics
}

\author{
Guang-Lei Wang, ${ }^{1}$ Lei Ying, ${ }^{1}$ Ying-Cheng Lai, ${ }^{1,2,3}$ and Celso Grebogi ${ }^{3}$ \\ ${ }^{1}$ School of Electrical, Computer, and Energy Engineering, Arizona State University, Tempe, Arizona 85287, USA \\ ${ }^{2}$ Department of Physics, Arizona State University, Tempe, Arizona 85287, USA \\ ${ }^{3}$ Institute for Complex Systems and Mathematical Biology, School of Natural and Computing Sciences, King's College, \\ University of Aberdeen, Aberdeen AB24 3UE, United Kingdom
}

(Received 14 January 2013; revised manuscript received 28 April 2013; published 15 May 2013)

\begin{abstract}
Quantum chaotic scattering is referred to as the study of quantum behaviors of open Hamiltonian systems that exhibit transient chaos in the classical limit. Traditionally a central issue in this field is how the elements of the scattering matrix or their functions fluctuate as a system parameter, e.g., the electron Fermi energy, is changed. A tacit hypothesis underlying previous works was that the underlying classical phase-space structure remains invariant as the parameter varies, so semiclassical theory can be used to explain various phenomena in quantum chaotic scattering. There are, however, experimental situations where the corresponding classical chaotic dynamics can change characteristically with some physical parameter. Multiple-terminal quantum dots are one such example where, when a magnetic field is present, the classical chaotic-scattering dynamics can change between being nonhyperbolic and being hyperbolic as the Fermi energy is changed continuously. For such systems semiclassical theory is inadequate to account for the characteristics of conductance fluctuations with the Fermi energy. To develop a general framework for quantum chaotic scattering associated with variable classical dynamics, we use multi-terminal graphene quantum-dot systems as a prototypical model. We find that significant conductance fluctuations occur with the Fermi energy even for fixed magnetic field strength, and the characteristics of the fluctuation patterns depend on the energy. We propose and validate that the statistical behaviors of the conductance-fluctuation patterns can be understood by the complex eigenvalue spectrum of the generalized, complex Hamiltonian of the system which includes self-energies resulted from the interactions between the device and the semi-infinite leads. As the Fermi energy is increased, complex eigenvalues with extremely smaller imaginary parts emerge, leading to sharp resonances in the conductance.
\end{abstract}

DOI: 10.1103/PhysRevE.87.052908

PACS number(s): 05.45.Mt, 73.23.-b, 73.63.Kv

\section{INTRODUCTION}

Quantum chaos is a field that explores the quantum manifestations of various chaotic behaviors of Hamiltonian systems in the classical limit [1-3]. In open Hamiltonian systems, a fundamental type of chaotic behaviors is transient chaos [4], which leads to chaotic scattering. Quantum chaotic scattering, referred to as the study of quantum behaviors caused by chaotic scattering in the corresponding classical system [5-9], is highly relevant to a number of fields in physics, such as atomic physics, condensed matter physics, and acoustics.

The major characteristic that distinguishes a quantum system from its classical counterpart is that in quantum mechanics, the system is characterized by a nonzero value of the Planck constant. Let $\hbar$ denote the Planck constant nondimensionalized by normalizing to characteristic length and momentum values, so $\hbar \rightarrow 0$ corresponds to the classical limit, $\hbar \ll 1$ to the semiclassical regime, and $\hbar \sim 1$ to the fully quantum mechanical regime. To study the quantum manifestation of classical Hamiltonian chaos, the semiclassical regime is of particular importance because this is the regime where both quantum and classical effects are relevant. Historically, of particular interest are signatures of chaotic scattering when the same system is treated quantum mechanically in the semiclassical regime [5-8].

The fundamental quantity characterizing a quantum scattering system is the scattering matrix, or the $S$ matrix, whose elements are the transition amplitudes between quantum states of the system before and after the scattering. The formulation of the $S$ matrix in terms of classical quantities had been of great interest in chemical physics even before chaos started attracting wide attention. The seminal contribution by Miller [10], who obtained a formula for $S$-matrix elements in terms of purely classical quantities in the semiclassical regime for reactive scattering systems, became the fundamental tool in the study of quantum chaotic scattering. Given a system that exhibits chaotic scattering in the classical limit, the $S$-matrix elements in the semiclassical regime exhibit random fluctuations as some physical parameters of the system, such as the energy of the scattering particle or the strength of some externally applied magnetic field, change in a classically small but quantum-mechanically large range. Depending on whether classical scattering is hyperbolic or nonhyperbolic [4], the statistical properties of the fluctuations in the $S$ matrix elements can be quite distinct [5-9]. In particular, the fluctuation patterns can be characterized by the energycorrelation function, where a faster decay of the function from unity as the energy difference is increased points to more severe fluctuation patterns. In their seminal work, Blümel and Smilansky [5] showed that the energy-correlation function is related to the decay law of trajectories in the classical phase space as Fourier-transform pairs. Thus, if classical trajectories decay faster from the scattering region, the energy-correlation function decreases more slowly from unity and vice versa.

A tacit assumption employed in previous works on quantum chaotic scattering is that, as a physical parameter such as the particle energy changes, the classical dynamics is invariant so the decay law remains unchanged, rendering meaningful its 
Fourier transform. Such situations arise in physical systems, for example, the transport of electrons through a quantum dot in the absence of magnetic field where the corresponding classical dynamics is essentially that of a open billiard and so does not depend on the electron energy. However, when a magnetic field is present, the characteristics of the classical dynamics can change drastically with the particle energy. For example, in an early work, Breymann, Kovács, and Tél studied the chaotic scattering of charged particles in an open three-disk billiard subject to a perpendicular magnetic field [11], which is effectively a three-terminal quantum-dot system in the presence of a magnetic field. Classically, since the Lorentz force depends on both the particle energy and the magnetic field, even for a fixed magnetic field strength the dynamics will depend on the energy. We thus face a challenge that, as the particle energy is systematically changed, there is no unique classical correspondence. Quantum mechanically, the scattering matrix elements will exhibit fluctuations with the particle energy, but the Fourier-transform formula relating the energy autocorrelation function and classical particle decay law is no longer applicable. How can then the fluctuation patterns be characterized and understood?

To investigate quantum chaotic scattering in lack of invariant classical dynamics, in this paper we use quantum-transport systems in two dimensions in the presence of a perpendicular magnetic field as a paradigm. To be concrete, we shall study multiple terminal (lead) graphene [12] quantum dots and focus on the conductance that is a function of scattering-matrix elements, as given by the Landauer formula [13,14]. For convenience, we call the quantum dot region excluding the semiinfinite leads the device region. In the terminology of scattering physics, such a region is called the scattering region, and we shall use both terms quite arbitrarily in this paper. For a fixed magnetic-field strength, as the Fermi energy of the conducting electrons increases, the underlying chaotic scattering dynamics in the classical limit can change its characteristics, and the quantum conductance can exhibit significant fluctuations. We find that the fluctuation patterns can be characterized and understood by the behaviors of the complex eigenvalues of the non-Hermitian Hamiltonian of the whole open system. In particular, when the leads are not present so the quantum-dot system is closed, the device Hamiltonian describing this closed system is Hermitian so all eigenenergies are real. The effect of a semi-infinite lead, however, can be treated by constructing the corresponding self-energy through the nonequilibrium Green's function (NEGF) formalism [14]. When all the self-energy terms corresponding to the multiple leads are combined with in the device Hamiltonian, the resulting Hamiltonian is no longer Hermitian but typically possesses complex eigenenergies. We shall demonstrate that, even for our situation where there is no unique classical dynamics, the magnitude of the imaginary parts of the eigenenergies provides a reasonable characterization of the conductance-fluctuation patterns. A particular feature is that, when eigenvalues with extremely small imaginary parts emerge, sharp conductance fluctuations occur in a relatively abrupt fashion over small energy scales.

It is important to place our theoretical approach in a proper context with respect to existing works in the quantum-chaotic scattering literature. We note that examining the poles of the $S$ matrix associated with the non-Hermitian effective
Hamiltonian of the interior system is the standard way to study quantum chaotic-scattering systems [15-19], which is effectively a random-matrix based approach. In particular, in the presence of a magnetic field where time-reversal symmetry is broken, the non-Hermitian Hamiltonian can be regarded as being drawn from the Gaussian unitary ensemble (GUE). The effective random-matrix type of Hamiltonian can be written as $H_{\text {eff }}=H-i \Gamma$, where $H$ is the Hermitian matrix describing the scattering region and $\Gamma$ describes the transition from the scattering region to the leads. The complex eigenvalues of $H_{\text {eff }}$ can then be used to characterize the poles of the $S$ matrix [15-19]. By performing the nonperturbative ensemble averaging one can obtain the statistical properties of the $S$ matrix, e.g., the distribution of resonances widths, phase shifts, and delay times, and so on. Especially, in the regime of wide, overlapping resonances, the $S$-matrix elements behave smoothly, while near a sharp resonance the $S$-matrix elements will change abruptly. Because of the universality of the random matrices in describing the quantum dynamics of classically chaotic systems, closed or open, the random-matrix based theory of quantum chaotic scattering is very general and can reveal universal statistical properties of the underlying system. The unique contribution of our work, however, lies in an explicit construction of the non-Hermitian Hamiltonian, e.g., the matrix $\Gamma$, in terms of the specific system under consideration. Especially, in our approach, the matrix $\Gamma$ is nothing but the self-energies that quantify the effects of the open leads on the otherwise closed Hermitian system through the nonequilibrium Green's functions, and such self-energy terms can be calculated explicitly when the scattering system is specified. Our approach, thus, is capable of yielding a concrete form of the non-Hermitian Hamiltonian with respect to any specific configuration of the scattering system, which offers tremendous advantage in experimental design and actual device development based on quantum chaotic-scattering dynamics. As we demonstrate using chaotic graphene systems under a perpendicular magnetic field, our approach is effective for understanding the fluctuation properties associated with quantum chaotic scattering even in the absence of invariant classical phase-space structure.

In Sec. II, we describe our model of multiterminal graphene quantum dots, study its classical phase-space structure, and briefly review the NEGF method for quantum transmission or conductance calculation. In Sec. III, we argue that the standard semiclassical treatment breaks down due to lack of invariant classical dynamics and then present an alternative theory based on the spectral properties of the non-Hermitian Hamiltonian of the open quantum-dot system. Numerical support is provided in Sec. IV. Concluding remarks are offered in Sec. V.

\section{MODEL}

\section{A. Classical dynamics}

Without loss of generality, we use the symmetric, threeterminal quantum-dot system as a prototypical model to address the issue of conductance fluctuations in lack of invariant classical dynamics. Classically, the system consists of a scattering region and three semi-infinite leads separated angularly by $2 \pi / 3$, as shown schematically in Fig. 1 . The scattering 


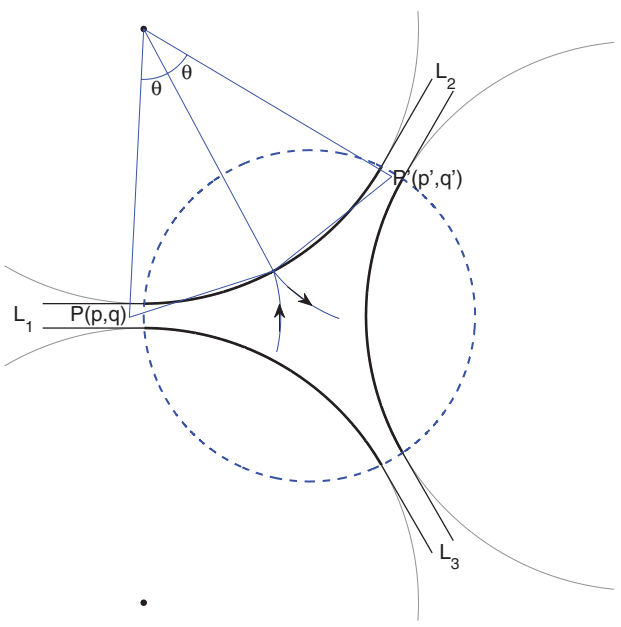

FIG. 1. (Color online) Geometry of a three-terminal quantum dot system. The boundary of the device is denoted by the black heavy lines. A perpendicular magnetic field is applied in the circular region defined by the blue dashed lines. The system is made entirely of graphene and the infinite ribbons have zigzag boundaries. The corresponding classical dynamics is effectively that of an open billiard system with three outgoing channels.

region is specified by the black heavy lines, the boundaries of which consist of three circular arcs, where the centers of the respective circles are located at the three corners of an equilateral triangle, the coordinates of which are $(-d / \sqrt{3}, d),(-d / \sqrt{3},-$ $d),(2 d / \sqrt{3}, 0)$, respectively. The radius of each circle is $r$. We assume that the quantum-dot system is made entirely of graphene, so we choose the geometric parameters $d$ and $r$ in terms of the lattice constant of graphene: $d=199 a_{0}$ and $r=110 \sqrt{3} a_{0}$, where $a_{0}=1.42 \AA$ is the distance between two nearest-neighbor carbon atoms in graphene [12]. The boundary walls are hard, rendering elastic scattering off the walls. The semi-infinite leads are also assumed to be made of graphene. A perpendicular magnetic field is uniformly applied in the circular region enclosed by the blue-dashed circle in Fig. 1, the radius of which is $d / \sqrt{3}$. Due to the dependence of the Lorentz force on the particle velocity and, hence, on the particle energy, the classical dynamics depends on the energy as well. This means that, for the quantum-dot system, as the Fermi energy is varied, the corresponding classical phase-space structure will change [11]. In classical simulations, the electron moves effectively in free space, so its energy is given by $E=\frac{1}{2} m v^{2}$ and the cyclotron radius is $r=m v / e B$, where $m$ is the vacuum mass of the electron and $v$ is the Fermi velocity.

For a fixed magnetic field and a given energy, the chaotic invariant set in the classical phase space can be numerically calculated, as follows. Without collision with the wall, the electron trajectory is a circle defined by the cyclotron radius. Let $(p, q)$ be the coordinates of the center of the circle, say, before a collision. After the collision, it is only necessary to calculate the new center coordinates in terms of those before the collision and the system parameters. Let $\left(x_{i}, y_{i}\right)$ be the center of the boundary circle on which the collision occurs. The angle between $(p, q)$ and the collision point on the wall with respect to $\left(x_{i}, y_{i}\right)$ can be written as $\theta=\theta\left(p, q, x_{i}, y_{i}\right)$. The center coordinates of the electron cyclotron orbit after the
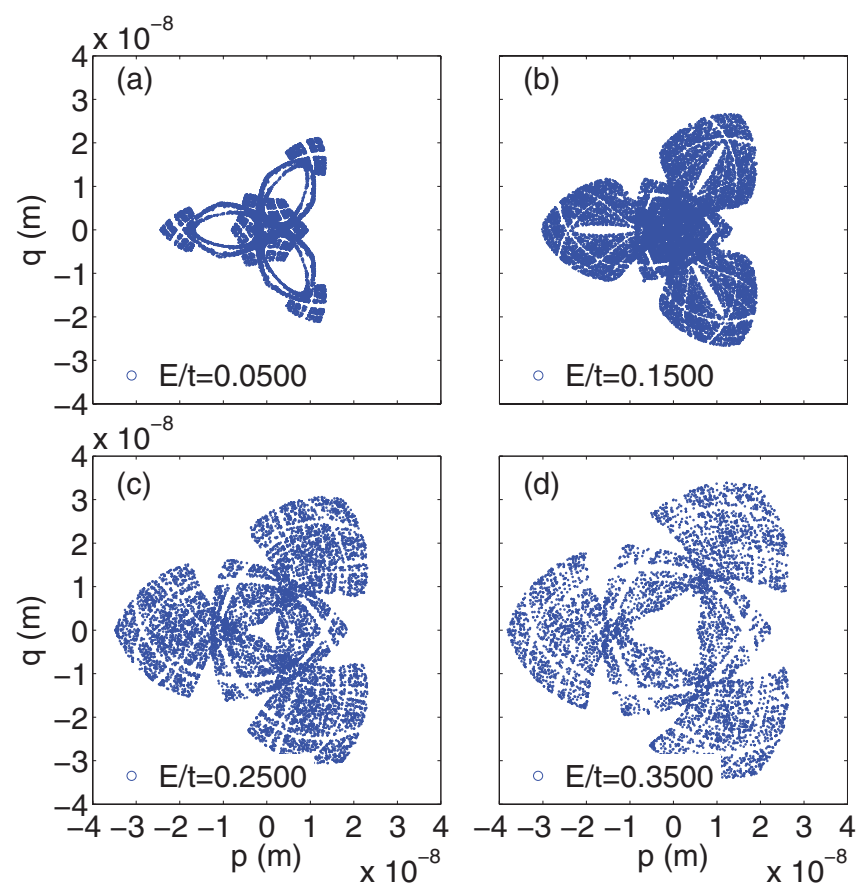

FIG. 2. (Color online) For fixed magnetic field specified by $\phi / \phi_{0}=0.0019$, representative chaotic sets for four different values of the energy: (a) $E / t=0.05$, (b) $E / t=0.15$, (c) $E / t=0.25$, and (d) $E / t=0.35$, where $p$ and $q$ are the coordinates of center points of the electron trajectory. We observe that the chaotic sets are characteristically distinct.

collision, $\left(p^{\prime}, q^{\prime}\right)$, can be determined by rotating the point $(p, q)$ with respect to $\left(x_{i}, y_{i}\right)$ by $2 \theta$ clockwise (or counterclockwise, depending on the direction of the magnetic field). In particular, we have

$$
\begin{aligned}
& p^{\prime}=x_{i}+\left(p-x_{i}\right) \cos (2 \beta)-\left(q-y_{i}\right) \sin (2 \beta), \\
& q^{\prime}=y_{i}+\left(p-x_{i}\right) \sin (2 \beta)+\left(q-y_{i}\right) \cos (2 \beta),
\end{aligned}
$$

where $\beta=\theta$ if the magnetic field is upward and $\beta=-\theta$ for downward magnetic field. Since the system is open, chaos is transient and the underlying chaotic invariant set is nonattracting, which can be calculated, for example, by using the sprinkler method [4]. Figure 2 shows, for a fixed magnetic field specified by the magnetic flux $\phi$, some representative chaotic sets for different values of the Fermi energy, where $\phi / \phi_{0}=0.0019$ and $\phi_{0}=h / e=4.136 \times 10^{-15} \mathrm{~T} \mathrm{~m}^{2}$ is the magnetic flux quanta. Here, for convenience of the quantum calculations, we use the magnetic flux quanta to normalize the magnetic flux. For graphene, the magnetic flux is $\phi=B S$, where $S=3 \sqrt{3} a_{0}^{2} / 2$ is the area of one honeycomb unit cell composed of six carbon atoms. For convenience, the electron Fermi energy is also normalized by the hopping energy in the tight-binding model of the graphene lattice, which is $t=$ $2.7 \mathrm{eV}$ [12].

\section{B. Calculation of quantum transmission and conductance}

We use the NEGF formalism [14] to calculate the conductance of the multiterminal quantum-dot system. The Green's 
function is defined as

$$
(E I-H) G(E)=I,
$$

where $H$ is the Hamiltonian of the whole system of device and semi-infinite leads, $E$ is the eigenvalue, and $I$ is the identity matrix. In order to calculate the various conductances between different pairs of leads, we need to calculate the Green's function of the device itself under the influence of the semi-infinite leads. The standard methodology is to introduce the non-Hermitian self-energy terms associated with the leads into Eq. (2). Let $H_{D}$ be the device Hamiltonian. For three terminals, the NEGF of the device, $G_{D}(E)$, can then be expressed by

$$
G_{D}(E)=\left[E I-H_{D}-\Sigma_{L_{1}}-\Sigma_{L_{2}}-\Sigma_{L_{3}}\right]^{-1},
$$

where $\Sigma_{L_{i}},(i=1,2,3)$ are the self-energy terms associated with the three leads, respectively.

For the graphene device, the tight-binding Hamiltonian is

$$
H_{D}=\sum_{\langle i, j\rangle}-t_{i j}\left(c_{i}^{\dagger} c_{j}+\text { H.c. }\right),
$$

where $t_{i j}=t$ is the hopping energy between the nearestneighbor sites of electrons, $\langle i, j\rangle$ indicates that only the nearest-neighbor pairs contribute to the whole energy, and $c_{i}^{\dagger}$ and $c_{i}$ are the creation and annihilation operators on site $i$, respectively. When a perpendicular uniform magnetic field is applied, the hopping energy is modified to

$$
t_{i j}=t e^{-i 2 \pi \phi_{i j}},
$$

where

$$
\phi_{i, j}=\frac{1}{\phi_{0}} \int_{j}^{i} \mathbf{A} \cdot d \mathbf{l}
$$

and $\mathbf{A}$ is the corresponding vector potential. At the hardwall boundaries of the device, as both the potential and its first derivative are infinite, the coupling elements in the Hamiltonian are zero. At the boundaries between the device and the leads, since the leads are also made of graphene, the coupling elements in the Hamiltonian matrix are finite and can be written down explicitly.

The self-energy terms $\Sigma_{L_{i}}$, which describe the effects of leads on the transport property through the device, can be solved self-consistently using a standard iteration procedure [20]. In particular, utilizing a recursive method (to be described below) to start from lead 1 (the "left" lead), we can calculate the surface retarded Green's functions of the three leads $\left(g_{L_{i}}\right)$ using

$$
\begin{aligned}
& g_{L_{i}}=\left[E^{+} I-H_{0}-H_{c}^{\dagger} \tilde{\Lambda}\right]^{-1} \quad i=1, \\
& g_{L_{i}}=\left[E^{+} I-H_{0}-H_{c} \Lambda\right]^{-1} i=2,3,
\end{aligned}
$$

where $E^{+}=E+i \eta\left(\eta \rightarrow 0^{+}\right), H_{0}$ is the Hamiltonian of one unit cell of the lead, $H_{c}$ is the coupling matrix between two neighboring unit cells, and $\Lambda$ and $\widetilde{\Lambda}$ are the so-called appropriate transfer matrices which can be calculated iteratively [14]. The self-energy functions $\Sigma_{L_{i}}(E)$ are given by

$$
\Sigma_{L_{i}}=H_{L_{i} D}^{\dagger} g_{L_{i}} H_{L_{i} D},
$$

where $H_{L_{i} D}$ is the coupling matrix between the $i$ th lead and the device. The size of $H_{L_{i} D}$ is $n_{L_{i}} \times N_{D}$, where $n_{L_{i}}$ is the number of atoms per unit cell of the $i$ th lead and $N_{D}$ is the total number of atoms in the device. Note that only the elements associated with the boundaries between the device and the leads are nonzero. The NEGF of the device can then be obtained from Eq. (3).

The quantum transmission of the three-terminal quantumdot system is given by $[14,20]$

$$
T_{i j}(E)=\operatorname{Tr}\left[\Gamma_{L_{i}} G_{D} \Gamma_{L_{j}} G_{D}^{\dagger}\right]
$$

where

$$
\Gamma_{L_{i}}=i\left[\Sigma_{L_{i}}-\Sigma_{L_{i}}^{\dagger}\right]
$$

and the subscript in $T_{i j}(E)$ indicates that the transmission is from the $j$ th lead to the $i$ th lead. Corresponding to the transmission $T_{i j}$, the conductance can be calculated by using the classic Landauer formula [13],

$$
G_{i j}(E)=\frac{2 e^{2}}{h} T_{i j}(E) .
$$

Due to rotational symmetry of the three-terminal quantumdot system, we discuss the transport properties between an arbitrary pair of leads, e.g., from lead 2 to lead 1, i.e., $T_{12}(E)$ and $G_{12}(E)$. The local density of states (LDS) at site $i$ can be obtained through

$$
n_{i}=-\frac{1}{\pi} \operatorname{Im}\left[G_{D}(i, i)\right],
$$

where $G_{D}(i, i)$ is the diagonal matrix element of the Green's function at the $i$ th site.

When the device is large, e.g., with tens of thousands of atoms, the device Hamiltonian matrix is also large and the required computation for the Green's function can become quite demanding. We use the recursive Green's function (RGF) method to calculate the transmission in a layer-by-layer manner. In particular, we divide the device into a large number of graphene layers and calculate the Green's function of each layer. In so doing the effect of all other layers and the leads are treated as some kind of self-energies. The advantage is basically to replace computations of large matrix by those associated with a large number of small matrices, leading to marked improvement in the computational efficiency.

\section{THEORY}

\section{A. Breakdown of semiclassical theory of quantum chaotic scattering in lack of invariant classical phase-space structure}

The fundamental quantity in the semiclassical theory of quantum chaotic scattering is the energy-correlation function of the $S$ matrix. Let $s(E)$ be an arbitrary element of the $S$ matrix $S(E)$, which depends on the Fermi energy $E$. The energycorrelation function is

$$
C(\Delta E)=\frac{\left\langle s^{*}(E) s(E+\Delta E)\right\rangle_{E}}{\left\langle|s(E)|^{2}\right\rangle},
$$

where $s^{*}(E)$ is the complex conjugate of $s(E),\langle\cdot\rangle_{E}$ denotes average over a classically small but quantum-mechanically large energy interval, say, $\Delta_{E}$, and $\Delta E \ll \Delta_{E}$ is a small energy increment. The seminal result of Blümel and Smilansky in their semiclassical treatment [5] is that $C(E)$ can be calculated by 
the Fourier transform of the classical particle-decay law $P(t)$,

$$
C(\Delta E) \sim \int P(t) e^{-i \Delta E t / \hbar} d t .
$$

For fully chaotic (hyperbolic) scattering, particle decay from the scattering region is exponential: $P(t) \sim e^{-\kappa t}$, where $\kappa$ is the escape rate associated with the underlying transient chaos [4]. Substituting the exponential-delay law into Eq. (13) gives a Lorentzian type of energy-correlation function $C(\Delta E)$, which is flat for $\Delta E \rightarrow 0$ [5]. For nonhyperbolic chaotic scattering where chaotic set and Kolmogorov-Arnold-Moser (KAM) islands coexist so the decay law is algebraic [21], $C(\Delta E)$ decreases fast from unity as $\Delta E$ is increased from zero [7].

We thus see that, while the semiclassical theory is powerful for revealing the quantum manifestations of classical chaotic scattering, Eq. (13) relies on the system's possessing a welldefined, unique particle decay law associated with the classical scattering dynamics as the particle energy is changed. For quantum-dot systems this is indeed the case in the absence of any magnetic field. However, as demonstrated in Sec. II A, when a magnetic field is present, the classical phase-space structure changes with the energy, as does the particle decay law. The formulation of the semiclassical theory of quantum chaotic scattering, Eq. (13), no longer holds.

\section{B. Spectral theory of quantum chaotic scattering in the presence of magnetic field}

A key quantity in determining the transmission or conductance through a quantum-dot system is the self-energy terms, which effectively reduce an infinite open system to a finite one. However, as can be seen from Eq. (7), the self-energy matrices $\Sigma_{L_{i}}(i=1,2,3)$ are not Hermitian, which is a consequence of the coupling between quantum states in the device and the continuum environment (leads) [22]. Nonetheless, the device Hamiltonian $H_{D}$ describes a closed system specified by the finite scattering region, as shown in Fig. 1, so it is Hermitian and defines the following eigenvalue problem:

$$
H_{D} \psi_{0 \alpha}=E_{0 \alpha} \psi_{0 \alpha},
$$

where the subscript $\alpha$ denotes the eigenvalue index and the eigenvalues $E_{0 \alpha}$ and the eigenfunctions $\psi_{0 \alpha}$ are all real. The time evolution of an eigenfunction is governed by

$$
i \hbar \frac{\partial}{\partial t} \psi_{0 \alpha}(t)=E_{0 \alpha} \psi_{0 \alpha}(t),
$$

for which the solution is

$$
\psi_{0 \alpha}(t)=\psi_{0 \alpha}(0) e^{-i \frac{E_{0 \alpha}}{\hbar} t} .
$$

For the entire open quantum-dot system where electrons go through the device from one lead to another, the Hamiltonian is not Hermitian. The eigenvalue problem can be written as

$$
\left[H_{D}+\Sigma_{L 1}+\Sigma_{L 2}+\Sigma_{L 3}\right] \psi_{\alpha}=E_{\alpha} \psi_{\alpha},
$$

where the eigenvalue $E_{\alpha}$ is complex and the eigenfunctions $\psi_{\alpha}$ no longer form an orthonormal set. We can express the energy as

$$
E_{\alpha}=E_{0 \alpha}-\triangle_{\alpha}-i \gamma_{\alpha},
$$

where $\triangle_{\alpha}$ represents the shift in the energy from the corresponding value in the closed device system as caused by the leads and $\gamma_{\alpha}$ is the imaginary part of the eigenvalue $E_{\alpha}$. In order to have a more quantitative expression for $\Delta_{\alpha}$ and $\gamma_{\alpha}$, we can treat the self-energy terms as small perturbation and keep only the first-order terms [9]. After some standard calculation we can obtain

$$
\triangle_{\alpha}+i \gamma_{\alpha} \approx-\left\langle\psi_{0 \alpha}\left|\Sigma_{L}\right| \psi_{0 \alpha}\right\rangle
$$

where $\Sigma_{L}=\Sigma_{L 1}+\Sigma_{L 2}+\Sigma_{L 3}$. We thus have

$$
\gamma_{\alpha} \approx-\operatorname{Im}\left(\left\langle\psi_{0 \alpha}\left|\Sigma_{L}\right| \psi_{0 \alpha}\right\rangle\right)=-\left\langle\psi_{0 \alpha}\left|\operatorname{Im}\left(\Sigma_{L}\right)\right| \psi_{0 \alpha}\right\rangle,
$$

which shows explicitly that the imaginary part of $E_{\alpha}$ is induced by the leads. Since the self-energy term $\Sigma_{L}$ represents the coupling between the device and leads, only the boundary parts of $\psi_{0 \alpha}$ contribute to the value of $\gamma_{\alpha}$. This means that the wave functions which describe delocalized states always have a stronger coupling with the leads than those describing strong localized states; that is, strongly localized states have much smaller $\gamma_{\alpha}$ values than delocalized states. In previous works, the quantity $\gamma_{\alpha}$ has been used in the study of conductance fluctuations [22-24].

Our idea is that, even when the classical phase-space structure varies with the Fermi energy (or any parameter, with respect to which conductance fluctuations are examined) so a semiclassical understanding is not available, the quantity $\gamma_{\alpha}$ can be used to characterize and understand the fluctuation patterns. To gain insights, it is useful to examine the origin of sharp resonances in the conductance-fluctuation curve for regular and chaotic dynamics in the classical limit. It has been known that sharp conductance fluctuations in open, nanoscale transport devices are typically caused by quantum pointer states, resonant states of finite but long lifetime formed inside the nanostructure $[25,26]$. These resonant states are in fact a kind of Fano resonance [27]. For example, for a quantum-dot system whose classical dynamics is regular or contains a significantly regular component, there are stable periodic orbits. If the dot geometry is closed, highly localized states can form around the periodic orbits, forming quantum scars [28]. When leads are attached to the quantum dot so the system becomes open, some periodic orbits can still survive, leading to resonant states or quantum pointer states. Since the corresponding classical orbits are stable, the resonant states can have long lifetime, leading to weak coupling with the leads. As a result, narrow resonances can form about the energy values that are effectively the eigenenergies in the corresponding closed system. When a modification to the dot geometry is introduced so the underlying classical dynamics becomes fully chaotic, no stable periodic orbits can exist. While scars can still be formed around classically unstable periodic orbits in the closed system [28] (even in graphene and Dirac fermion systems [29]), the corresponding resonant states in the open system generally will have much shorter lifetimes, effectively eliminating the narrow resonances in the conductance fluctuation pattern.

The interplay between conductance fluctuations and quantum pointer states, as discussed above, does not require an invariant classical phase-space structure. Consequently, the emergence of pointer states, mathematically characterized by relatively small values of the quantity $\gamma_{\alpha}$, provides a reasonable way to understand the conductance-fluctuation patterns for quantum-dot systems in the presence of a magnetic field. 


\section{RESULTS}

Figure 2 shows that, in the presence of a magnetic field of flux strength $\phi / \phi_{0}=0.0019$, as the Fermi energy of the electron is increased, the underlying chaotic set in the classical limit changes markedly. The characteristics of the conductance-fluctuation patterns then depend on the energy value. An example is shown in Fig. 3, where we observe quite distinct behaviors of the conductance fluctuations. For instance, for energy values in a small interval about $E / t=$ 0.05 [Fig. 3(a)], the normalized conductance is near unity and there is hardly any fluctuation. However, for energy values about, say, $E / t=0.25$, there are sharp, resonance-type of variations in the conductance, as shown in Fig. 3(c). We notice that the corresponding chaotic invariant set for $E / t=0.25$ is much more extensive in the phase space than that associated with $E / t=0.05$. In fact, for high energy values, there are large-size KAM islands in the phase space [11].

We now demonstrate that the local conductance-fluctuation patterns exemplified in Figs. 3(a)-3(d) can be understood through the imaginary part of the complex energy eigenvalue, $\gamma_{\alpha}$, as discussed in Sec. III. For clarity, we plot $\gamma_{\alpha}$ versus $\operatorname{Re}\left(E_{\alpha}\right)$ in units of $t$, where $\gamma_{\alpha} / t$ is on a logarithmic scale, as shown in Fig. 4. We notice the following three features: (i) the range of variation in $\gamma_{\alpha} / t$ is over six orders of magnitude, (ii) a substantial fraction of large values of $\gamma_{\alpha} / t$ appear to follow a curve that plateaus approximately for $\gamma_{\alpha} / t \lesssim 10^{-2}$, and (iii) the eigenvalues with smaller imaginary parts begin to appear for $E / t \approx 0.15$.

Mathematically, the imaginary part of the complex eigenenergy contributes an exponential decay factor to the wave function. Physically, this means that the electrons entering the device from one lead will eventually scatter out of the device into leads, and the imaginary part of the eigenvalue is effectively the inverse of the lifetime of electrons in a particular state in the device [14]. Another physical significance of $\gamma_{\alpha}$ is that it characterizes the width of Fano resonance that occurs

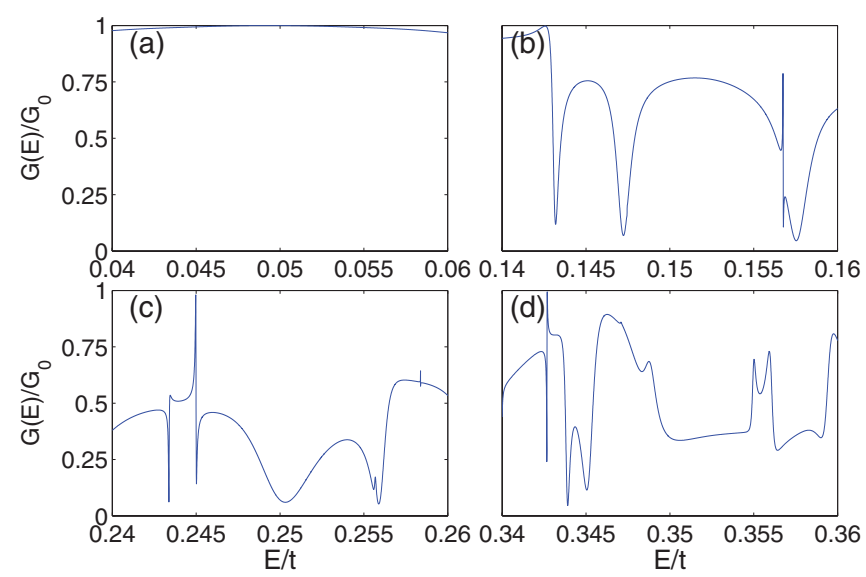

FIG. 3. (Color online) [(a)-(d)] Normalized conductance (by $\left.G_{0}=2 e^{2} / h\right)$ versus the Fermi energy for our three-terminal graphene quantum-dot system for small energy intervals around $E / t=0.05$, $0.15,0.25$, and 0.35 , respectively. The conductance-fluctuation patterns differ markedly for different energy values, due to the characteristic difference in the corresponding phase-space structure (Fig. 2).

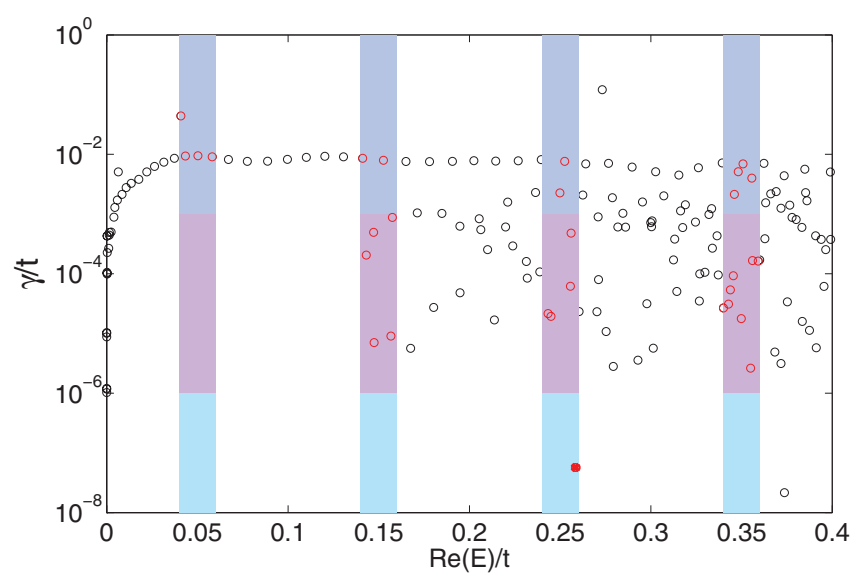

FIG. 4. (Color online) Imaginary part $\gamma_{\alpha}$ of the complex eigenvalue of the non-Hermitian Hamiltonian for the three-terminal graphene quantum-dot system versus the real part, where $\gamma_{\alpha}$ is represented on a logarithmic scale. The magnetic flux is $\phi / \phi_{0}=$ 0.0019. The four vertical bands correspond to the four cases of conductance fluctuations in Figs. 3(a)-3(d), respectively. For the first band around $E / t=0.05$, the values of $\gamma_{\alpha}$ are all large (about $10^{-2}$ ), giving rise to the smooth conductance variation in Fig. 3(a). Much smaller values of $\gamma_{\alpha}$ appear in the remaining three vertical bands, leading to significant conductance fluctuations as in Figs. 3(b)-3(d). The three colors in each band specify different ranges of the values of $\gamma_{\alpha} / t$.

commonly in quantum-dot systems [22]. From Fig. 3, we can see that there are two kinds of fluctuations: smooth variations and sharp fluctuations. The smooth variations are caused by fully chaotic scattering and the values of the corresponding transmission into all leads are of the order of unity, due to coherent superposition of overlapping resonances. In terms of the complex eigenenergy, the transmission in this case corresponds to some poles with large imaginary parts. The sharp fluctuations are caused by the strongly localized states in the devices, for example, Fano resonances. The coupling between such a strongly localized state and the leads is in general quite weak so the imaginary part of $E_{\alpha} / t$ becomes extremely small, typically on the order $10^{-3} \sim 10^{-8}$. To verify these features, we have calculated the LDS patterns for a large number of energy values. For smooth transmission variations, the LDS patterns are typically not localized in the scattering region, as exemplified in Fig. 5(a). In fact, in this case, boundary states prevail, a typical feature of graphene [12]. However, for sharp fluctuations in the transmission, highly localized LDS patterns are common, examples of which are shown in Figs. 5(b)-5(d).

We note that previous random-matrix theory predicts that, in a quantum chaotic scattering system, the range of the statistical distribution range of the imaginary part of the eigenenergy $E$ is broadened with increase in the real part [15]. Our results, such as those in Fig. 4, are consistent with this prediction. We also note that, in the presence of a vertical magnetic field, certain sharp fluctuations of the $S$-matrix elements with energy can be related to classical unstable periodic orbits [16].

The weak coupling between discrete states in the device with continuum in the open leads, however, is not the only condition under which Fano resonances may be observed. The 
(a)

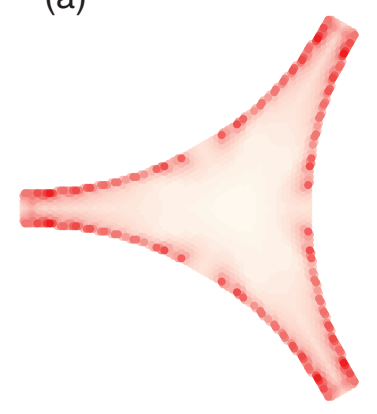

(b)

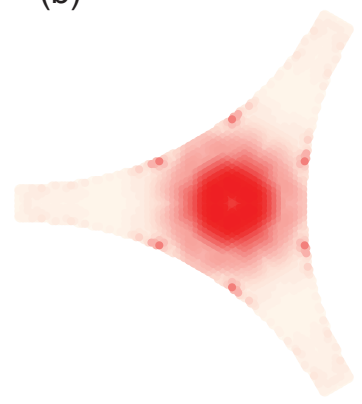

(c)

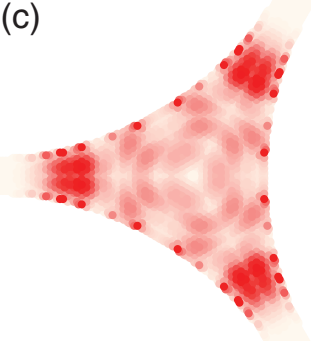

FIG. 5. (Color online) Local density of states in the three-terminal graphene quantum-dot system for different Fermi energies: (a) $E / t=$ 0.05 , (b) $E / t=0.1474$, (c) $E / t=0.245$, and (d) $E / t=0.355$.

Fermi energy of electrons has to reach a threshold to activate the first level of the discrete system (closed device), which can be seen in the presence of a magnetic field because such levels are in fact the Landau levels of the system [30]. From Fig. 4, for example, extremely small values of $\gamma_{\alpha}$ start to appear around $\operatorname{Re}\left(E_{\alpha}\right) / t=0.15$, which is caused by the emergence of the first Landau level about that energy. The pattern in Fig. 5(b) is in fact one associated with the first Landau level at energy $E / t=0.1474$. As the energy is increased through the second and third Landau levels, the physics remains the same even though the scattering process becomes more complicated due to chaos, phases change of paths, their superpositions, and so on. These results indicate that the values of the imaginary parts of the complex eigenenergies of the non-Hermitian Hamiltonian explain well the conductance-fluctuation patterns in the presence of a magnetic field, even when the underlying classical dynamics varies with the Fermi energy.

To demonstrate the generality of using the complex eigenenergies of the non-Hermitian Hamiltonian to characterize conductance fluctuations in the absence of classically invariant phase-space structure, we present results with a four-terminal graphene quantum-dot system, where the left and right leads have zigzag edges, and the upper and down leads have armchair edges, as shown in Fig. 6. In particular, the geometry of the system is shown in Fig. 6(a), where the scattering (device) region is denoted by thick black curves and the region in which the perpendicular magnetic field is applied is represented by the blue dashed circle. Figure 6(b) shows the values of the eigenenergies in the complex plane, where we see that for $E>0$, eigenvalues with extremaly small imaginary parts begin to appear after the first Landau level
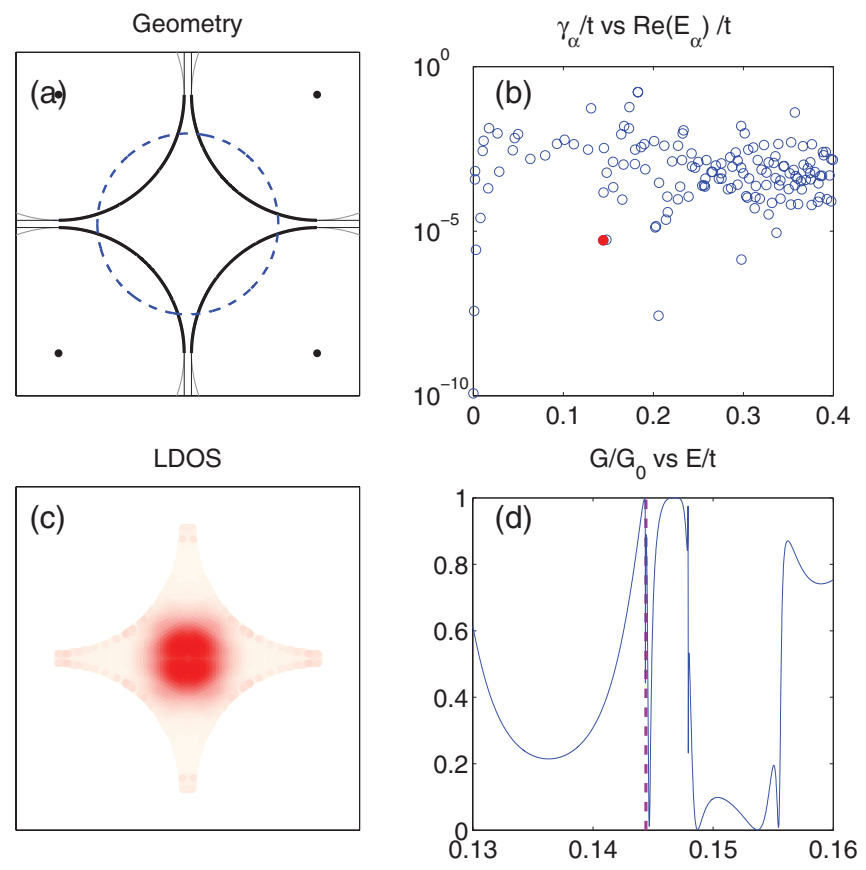

FIG. 6. (Color online) Conductance fluctuations and characterization in a four-terminal graphene quantum-dot system under a perpendicular magnetic field of flux $\phi / \phi_{0}=0.0019$. (a) Geometry of the system (same legend as in Fig. 1), (b) complex eigenvalue spectrum, (c) localized density of states for $E / t=0.1444$, (d) Local conductance fluctuations from the left to the upper leads about the first Landau level, i.e., $E / t=0.1444$. The filled red circle in (b) and the violet dashed line in (d) correspond to $E / t=0.1444$.

arises for $E / t \gtrsim 0.13$. A representative strongly localized state, corresponding to the filled red circle in Fig. 6(b), is shown in Fig. 6(c), whose coupling with the leads is weak. The corresponding local conductance-fluctuation pattern is shown in Fig. 6(d), where the vertical red dashed line corresponds to the real energy value of the filled red circle in Fig. 6(b). Overall, we observe similar behaviors to those in the three-terminal system.

\section{CONCLUSION}

We have argued that a foundational result in the conventional semiclassical theory of quantum chaotic scattering, namely the formula of the energy autocorrelation function for the $S$-matrix elements, breaks down when a magnetic field is present, for example, in a multiterminal quantum-dot system. The basic reason is that the semiclassical formula relies on the assumption that the corresponding classical chaotic set, or more broadly the classical phase-space structure, remains unchanged in the range where the energy is varied. However, under a magnetic field the classical phase-space structure depends on the energy [11]. The main contribution of this paper is an alternative framework to understand quantum chaotic scattering in situations where the classical phase-space structure varies with energy (or any other physical parameter, with respect to which the fluctuations of the $S$-matrix elements are probed).

Utilizing multiterminal graphene quantum dots as a prototypical class of systems, we articulate that quantum chaotic 
scattering can be physically characterized by the complex eigenvalues of the non-Hermitian Hamiltonian of the underlying system. A quantum-dot system can in general be decomposed into two parts: a closed device or scattering region and the set of semi-infinite electronic waveguides (or leads). The device Hamiltonian is Hermitian and permits a discrete set of eigenvalues, but the leads possess a continuum of energy spectrum mathematically described by complex self-energy terms. Adding the self-energies to the device Hamiltonian leads to a non-Hermitian Hamiltonian describing the entire open quantum system, whose eigenvalues are, in general, complex. The key physics to quantum chaotic scattering is that the inverse of the imaginary part of a complex eigenvalue is nothing but the lifetime of the corresponding eigenstate in the scattering region. Thus, when an energy eigenvalue possesses an extremely small imaginary part, the corresponding eigenstate has a long lifetime, which is effectively a strongly localized state. The coupling between such a localized state and the leads, or the quantum "environment" of the device, must necessarily be weak. This leads to an abrupt change in the transmission or conductance over an extremely small energy scale, the so-called Fano resonance [26,27]. Overall, in order to explain a numerically calculated or experimentally observed conductance-fluctuation pattern in quantum-dot systems under a magnetic field, one can calculate the complex eigenenergies of the corresponding non-Hermitian Hamiltonian. Energy regions in which the eigenenergies exhibit small imaginary parts are the regions where severe conductance fluctuations can be anticipated.

\section{ACKNOWLEDGMENTS}

This work was supported by AFOSR under Grant No. FA9550-12-1-0095 and by ONR under Grant No. N0001408-1-0627.
[1] M. C. Gutzwiller, Chaos in Classical and Quantum Mechanics (Springer, New York, 1990).

[2] H. J. Stöckmann, Quantum Chaos: An Introduction (Cambridge University Press, Cambridge, England, 1999).

[3] F. Haake, Quantum Signatures of Chaos, 2nd ed. (Springer, Berlin, 2001).

[4] Y.-C. Lai and T. Tél, Transient Chaos: Complex Dynamics on Finite-Time Scales (Springer, New York, 2011).

[5] R. Blümel and U. Smilansky, Phys. Rev. Lett. 60, 477 (1988); Physica D 36, 111 (1989).

[6] R. A. Jalabert, H. U. Baranger, and A. D. Stone, Phys. Rev. Lett. 65, 2442 (1990); C. M. Marcus, A. J. Rimberg, R. M. Westervelt, P. F. Hopkins, and A. C. Gossard, ibid. 69, 506 (1992).

[7] Y.-C. Lai, R. Blümel, E. Ott, and C. Grebogi, Phys. Rev. Lett. 68, 3491 (1992); A. P. S. deMoura, Y. C. Lai, R. Akis, J. P. Bird, and D. K. Ferry, ibid. 88, 236804 (2002).

[8] R. Ketzmerick, Phys. Rev. B 54, 10841 (1996); R. P. Taylor, R. Newbury, A. S. Sachrajda, Y. Feng, P. T. Coleridge, C. Dettmann, N. Zhu, H. Guo, A. Delage, P. J. Kelly, and Z. Wasilewski, Phys. Rev. Lett. 78, 1952 (1997); A. S. Sachrajda, R. Ketzmerick, C. Gould, Y. Feng, P. J. Kelly, A. Delage, and Z. Wasilewski, ibid. 80, 1948 (1998); B. Huckestein, R. Ketzmerick, and C. H. Lewenkopf, ibid. 84, 5504 (2000); G. Casati, I. Guarneri, and G. Maspero, ibid. 84, 63 (2000); R. Crook, C. G. Smith, A. C. Graham, I. Farrer, H. E. Beere, and D. A. Ritchie, ibid. 91, 246803 (2003).

[9] R. Yang, L. Huang, Y.-C. Lai, and C. Grebogi, Europhys. Lett. 94, 40004 (2011).

[10] W. H. Miller, Adv. Chem. Phys. 30, 77 (1975).

[11] W. Breymann, Z. Kovacs, and T. Tel, Phys. Rev. E 50, 1994 (1994).

[12] K. S. Novoselov, A. K. Geim, S. V. Morozov, D. Jiang, Y. Zhang, S. V. Dubonos, I. V. Grigorieva, and A. A. Firsov, Science 306, 666 (2004); C. Berger, Z. Song, T. Li, X. Li, A. Y. Ogbazghi, R. Feng, Z. Dai, A. N. Marchenkov, E. H. Conrad, P. N. First, and W. A. de Heer, J. Phys. Chem. B 108, 19912 (2004); C. W. J. Beenakker, Rev. Mod. Phys. 80, 1337 (2008); A. H. Castro Neto,
F. Guinea, N. M. R. Peres, K. S. Novoselov, and A. K. Geim, ibid. 81, 109 (2009); S. Das Sarma, S. Adam, E. H. Hwang, and E. Rossi, ibid. 83, 407 (2011).

[13] R. Landauer, Phil. Mag. 21, 863 (1970).

[14] S. Datta, Electronic Transport in Mesoscopic Systems (Cambridge University Press, Cambridge, UK, 1995).

[15] W. John, B. Milek, H. Schanz, and P. Seba, Phys. Rev. Lett. 67, 1949 (1991).

[16] Y. V. Fyodorov and H.-J. Sommers, J. Math. Phys. 38, 1918 (1997).

[17] Y. V. Fyodorov and B. A. Khoruzhenko, Phys. Rev. Lett. 83, 65 (1999).

[18] Y. V. Fyodorov and H.-J. Sommers, J. Phys. A: Math. Gen. 36, 3303 (2003).

[19] A. Ossipov and Y. V. Fyodorov, Phys. Rev. B 71, 125133 (2005).

[20] T. C. Li and S.-P. Lu, Phys. Rev. B 77, 085408 (2008); L. Huang, Y.-C. Lai, D. K. Ferry, R. Akis, and S. M. Goodnick, J. Phys.: Condens. Matter 21, 344203 (2009).

[21] See, for example, J. D. Meiss, J. R. Cary, C. Grebogi, J. D. Crawford, A. N. Kaufman, and H. D. I. Abarbanel, Physica D 6, 375 (1983); B. V. Chirikov and D. L. Shepelyansky, ibid. 13, 395 (1984); J. D. Meiss and E. Ott, ibid. 20, 387 (1986); A. Bambini and E. J. Robinson, Phys. Rev. A 45, 4661 (1992); G. Cristadoro and R. Ketzmerick, Phys. Rev. Lett. 100, 184101 (2008).

[22] M. Mendoza, P. A. Schulz, R. O. Vallejos, and C. H. Lewenkopf, Phys. Rev. B 77, 155307 (2008).

[23] I. Rotter, J. Phys. A: Math. Theor. 42, 153001 (2009).

[24] R. Yang, L. Huang, Y.-C. Lai, and L. M. Pecora, Appl. Phys. Lett. 100, 093105 (2012).

[25] W. H. Zurek, Rev. Mod. Phys. 75, 715 (2003); Nat. Phys. 5, 181 (2009); M. Zwolak, H. T. Quan, and W. H. Zurek, Phys. Rev. Lett. 103, 110402 (2009).

[26] D. K. Ferry, R. Akis, and J. P. Bird, Phys. Rev. Lett. 93, 026803 (2004); R. Brunner, R. Akis, D. K. Ferry, F. Kuchar, and R. Meisels, ibid. 101, 024102 (2008); A. M. Burke, R. Akis, T. E. Day, Gil Speyer, D. K. Ferry, and B. R. Bennett, ibid. 104, 176801 (2010). 
[27] U. Fano, Phys. Rev. 124, 1866 (1961).

[28] S. W. McDonald and A. N. Kaufman, Phys. Rev. Lett. 42, 1189 (1979); Phys. Rev. A 37, 3067 (1988); E. J. Heller, Phys. Rev. Lett. 53, 1515 (1984); E. B. Bogomolny, Physica D 31, 169 (1988); M. V. Berry, Proc. Roy. Soc. (London) A 423, 219 (1989).
[29] L. Huang, Y.-C. Lai, D. K. Ferry, S. M. Goodnick, and R. Akis, Phys. Rev. Lett. 103, 054101 (2009); X. Ni, L. Huang, Y.-C. Lai, and C. Grebogi, Phys. Rev. E 86, 016702 (2012); H.-Y. Xu, L. Huang, Y.-C. Lai, and C. Grebogi, Phys. Rev. Lett. 110, 064102 (2013).

[30] L. Ying, L. Huang, Y.-C. Lai, and C. Grebogi, Phys. Rev. B 85, 245448 (2012). 